Article

\title{
Rapid, Sensitive, and Accurate Point-of-Care Detection of Lethal Amatoxins in Urine
}

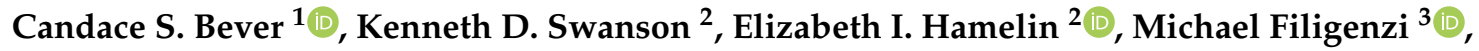 \\ Robert H. Poppenga ${ }^{3}$, Jennifer Kaae ${ }^{4}$, Luisa W. Cheng ${ }^{1, *}$ and Larry H. Stanker ${ }^{1}$ \\ 1 Foodborne Toxin Detection and Prevention Research Unit, Western Regional Research Center, Agricultural \\ Research Service, United States Department of Agriculture, 800 Buchanan Street, Albany, CA 94710, USA; \\ candace.bever@usda.gov (C.S.B.); lstanker@gmail.com (L.H.S.) \\ 2 Division of Laboratory Sciences, National Center for Environmental Health, Centers for Disease Control and \\ Prevention, Atlanta, GA 30333, USA; ost3@cdc.gov (K.D.S.); ehamelin@cdc.gov (E.I.H.) \\ 3 California Animal Health and Food Safety Laboratory System, University of California, 620 West Health \\ Sciences Drive, Davis, CA 95616, USA; msfiligenzi@ucdavis.edu (M.F.); rhpoppenga@ucdavis.edu (R.H.P.) \\ 4 Pet Emergency and Specialty Center of Marin, 901 E. Francisco Blvd, San Rafael, CA 94901, USA; \\ jenkaae@gmail.com \\ * Correspondence: luisa.cheng@usda.gov; Tel.: +1-510-559-6337
}

Received: 30 January 2020; Accepted: 12 February 2020; Published: 15 February 2020

\begin{abstract}
Globally, mushroom poisonings cause about 100 human deaths each year, with thousands of people requiring medical assistance. Dogs are also susceptible to mushroom poisonings and require medical assistance. Cyclopeptides, and more specifically amanitins (or amatoxins, here), are the mushroom poison that causes the majority of these deaths. Current methods (predominantly chromatographic, as well as antibody-based) of detecting amatoxins are time-consuming and require expensive equipment. In this work, we demonstrate the utility of the lateral flow immunoassay (LFIA) for the rapid detection of amatoxins in urine samples. The LFIA detects as little as $10 \mathrm{ng} / \mathrm{mL}$ of $\alpha$-amanitin ( $\alpha$-AMA) or $\gamma$-AMA, and $100 \mathrm{ng} / \mathrm{mL}$ of $\beta$-AMA in urine matrices. To demonstrate application of this LFIA for urine analysis, this study examined fortified human urine samples and urine collected from exposed dogs. Urine is sampled directly without the need for any pretreatment, detection from urine is completed in $10 \mathrm{~min}$, and the results are read by eye, without the need for specialized equipment. Analysis of both fortified human urine samples and urine samples collected from intoxicated dogs using the LFIA correlated well with liquid chromatography-mass spectrometry (LC-MS) methods.
\end{abstract}

Keywords: lateral flow immunoassay; amatoxins; amanitins; point-of-care; mushroom poisoning

Key Contribution: The study demonstrates the utility of the lateral flow immunoassay for the rapid detection of amatoxins in urine.

\section{Introduction}

Distinguishing toxic mushrooms from non-toxic ones is highly challenging, even for expert mycologists. Techniques to properly identify a mushroom include detailed morphological examination of the mushroom body, substrate identification, and knowledge of the location and the season. The toxins often associated with lethal cases are cyclopeptides, and more specifically amanitins (most commonly $\alpha$-amanitin ( $\alpha$-AMA), $\beta$-AMA, and $\gamma$-AMA, collectively referred to as amatoxins) [1] (Figure 1). Amatoxins are found in a few species of mushrooms from different genera, including Amanita, Galerina, and Lepiota [2]. Amatoxins are highly resistant to degradation, and on the cellular 
level they inhibit transcription by binding to RNA polymerase II. As little as $0.1 \mathrm{mg} / \mathrm{kg}$ body weight of amatoxins may cause death [3,4], and this amount can be found in a single Amanita phalloides.

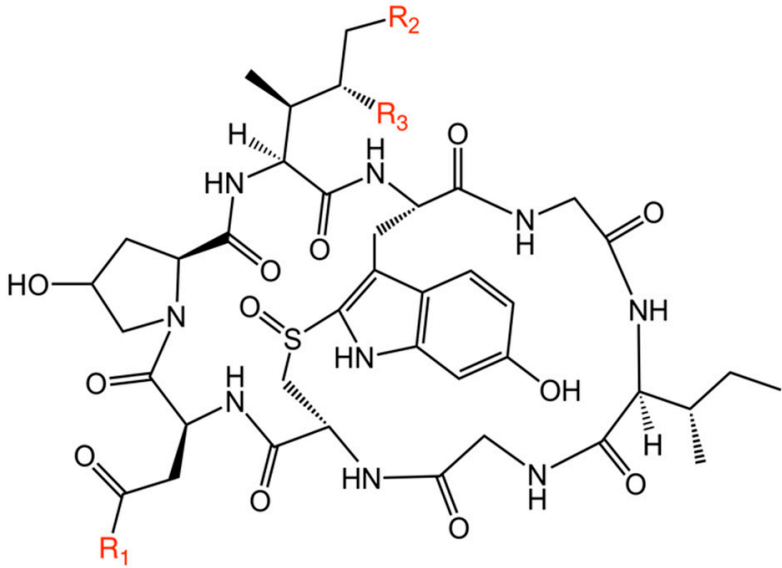

(a)

\begin{tabular}{cccc}
\hline Compound & $\mathbf{R}_{\mathbf{1}}$ & $\mathbf{R}_{\mathbf{2}}$ & $\mathbf{R}_{\mathbf{3}}$ \\
\hline$\alpha$-amanitin & $\mathrm{NH}_{2}$ & $\mathrm{OH}$ & $\mathrm{OH}$ \\
\hline$\beta$-amanitin & $\mathrm{OH}$ & $\mathrm{OH}$ & $\mathrm{OH}$ \\
\hline$\gamma$-amanitin & $\mathrm{NH}_{2}$ & $\mathrm{OH}$ & $\mathrm{H}$ \\
\hline
\end{tabular}

(b)

Figure 1. Chemical structures of the amatoxin variants examined in this paper, (a) molecular structure of the amanitin, (b) R-group designations for each variant.

Consumption of toxin-containing mushrooms can result in a range of symptoms, from mild to life-threatening [5,6]. The presumptive diagnosis for amatoxin poisoning is based on a history of consuming wild mushrooms (if known), presentation of delayed gastroenteritis, elevated liver enzyme levels, and ruling out other gastrointestinal diseases or conditions [6]. To distinguish amatoxin poisonings, the presence of an amatoxin in an intoxicated patient's urine would provide a definitive diagnosis. For dogs, obtaining a history of mushroom ingestion is rare, making diagnosis even more challenging. There are only a few laboratories capable of testing biological specimens for amatoxins to confirm human or animal exposures, and even when available, test results might not be available soon enough to help guide treatment. Although there are no FDA-approved antidotes, early diagnosis, aggressive immediate supportive care, and a range of potential therapies can potentially improve patient outcomes [6-11].

For both humans and dogs, the first symptoms of amatoxin poisonings usually appear 6-24 h after ingestion of an amatoxin-containing mushroom [6]. By this time, amatoxins have already begun damaging the liver and kidneys. Based on toxicological studies, amatoxins disappear rapidly from the serum, but are detectable in urine up to 4 days after ingestion [12-14]. In human urine, toxin concentrations decreased over time, and the highest concentrations observed were $4820 \mathrm{ng} / \mathrm{mL}$ for $\alpha$-AMA and $7103 \mathrm{ng} / \mathrm{mL}$ for $\beta$-AMA [12]. Because of the relative ease of obtaining a urine sample, and the longer duration of detectability of amatoxins in urine compared to serum, urine seems an obvious sample matrix for performing rapid amatoxin analysis. Sensitive, rapid, and easy-to-perform methods are needed to detect amatoxins for the early diagnosis of toxin poisoning $[9,15,16]$.

Current methods of chemical detection of amatoxins in urine include liquid chromatography-mass spectrometry (LC-MS) methods [17-23] and antibody-based enzyme-linked immunosorbent assays (ELISAs) [24,25]. LC-MS methods require sample extraction and expensive equipment, while ELISA methods require specialized equipment. Methods of both types typically take a few hours to complete. Lateral flow immunoassay (LFIA) formats utilize some of the reagents used for ELISA, but the entire test can be completed in minutes and requires no specialized equipment.

We have recently developed an LFIA for the detection of mushroom amatoxins [26]. As the sensitivity of the LFIA allows it to detect as little as $10 \mathrm{ng} / \mathrm{mL}$, we hypothesized that this test would be useful for urine analysis in instances of mushroom poisonings. To test this hypothesis, we first conducted analysis of urine samples that were fortified with toxins (blind to the analyst) based on 
the reported concentrations of amatoxins identified in exposed individuals; and second, we used the LFIA to detect toxins in the urine samples collected from poisoned dogs. The LFIA results were compared to the established LC-MS methods $[17,20]$. Based on these studies, we can begin identifying the diagnostic utility of the LFIA for identifying amatoxin exposure.

\section{Results and Discussion}

\subsection{Analytical Sensitivity of the LFIA and Interpretation of Results}

Standard curves were obtained for the detection of $\alpha$-AMA, $\beta$-AMA, and $\gamma$-AMA in a pooled urine matrix (Figure 2). As this is a competitive binding assay, the test line signal intensity decreases with the increase in toxin concentrations. The cut-off value for each individual amanitin was determined by selecting the concentration where the test line almost completely disappears, which is equivalent to a pixel intensity value of approximately 30. For $\alpha$-AMA and $\gamma$-AMA, the cut-off value is $10 \mathrm{ng} / \mathrm{mL}$, while for $\beta$-AMA, the cut-off is $100 \mathrm{ng} / \mathrm{mL}$. Although difficult to discern by eye, the limit of detection (defined as three times the standard deviation of a sample without amatoxins) is $0.3 \mathrm{ng} / \mathrm{mL}$ for $\alpha$-AMA and $\gamma$-AMA and $1 \mathrm{ng} / \mathrm{mL}$ for $\beta$-AMA. To ensure consistent interpretation of the line intensity by eye, the cut-off values determined in this study were used for the remainder of this study to determine the diagnostic accuracy of the test for urine analysis.

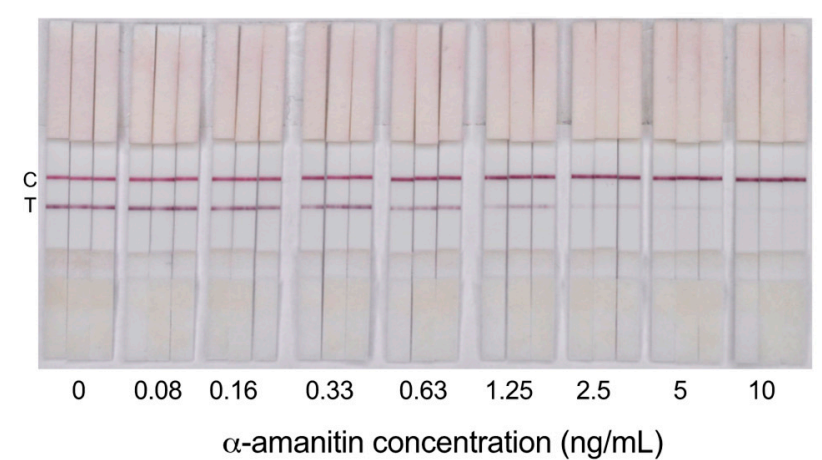

(a)

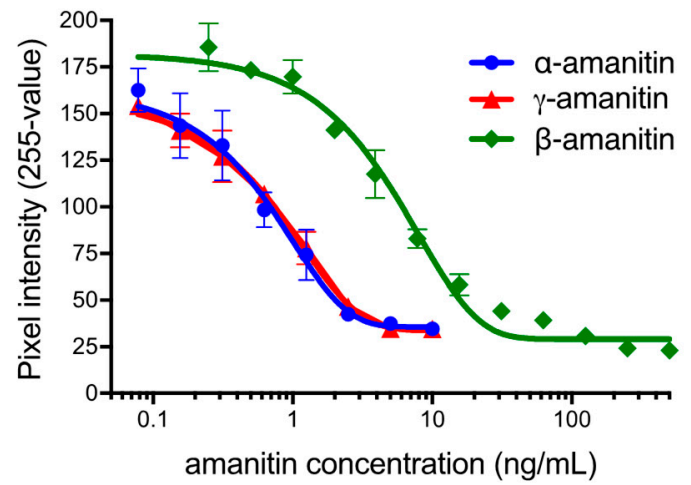

(b)

Figure 2. Standard curves for the detection of amatoxins by the lateral flow immunoassay (LFIA) in a pooled urine matrix. (a) A representative visual image of the LFIA test strips used for detecting $\alpha$-amanitin ( $\alpha$-AMA). (b) Digitized values for the test line intensity for the detection of $\alpha$-AMA, $\beta$-AMA, and $\gamma$-AMA. Data points represent the average of three replicates with error bars. T: test line, C: control line.

The cut-off value for $\beta$-AMA when detected in urine resulted in a 20-fold more sensitive assay cut-off value than our previous standard curve developed in phosphate-buffered saline (PBS) [26]. There was no change in sensitivity for $\alpha$-AMA or $\gamma$-AMA when using urine or PBS as the matrix [26]. To test whether the $\beta$-AMA sensitivity differences were possibly due to the $\mathrm{pH}$ of the matrix, we evaluated $\mathrm{pH}$-adjusted PBS buffers ranging from 4.5 to 8 (Figure 3 ). Indeed, there was a positive trend for the two concentrations of $\beta$-AMA tested (100 and $25 \mathrm{ng} / \mathrm{mL}$ ), in which the test line intensity increased with the increase in $\mathrm{pH}$. This trend corroborated with the differences between the $\mathrm{pH}$ of urine and that of PBS. The $\mathrm{pH}$ of the pooled urine matrix was 6.0, while the $\mathrm{pH}$ of PBS was 7.4. 


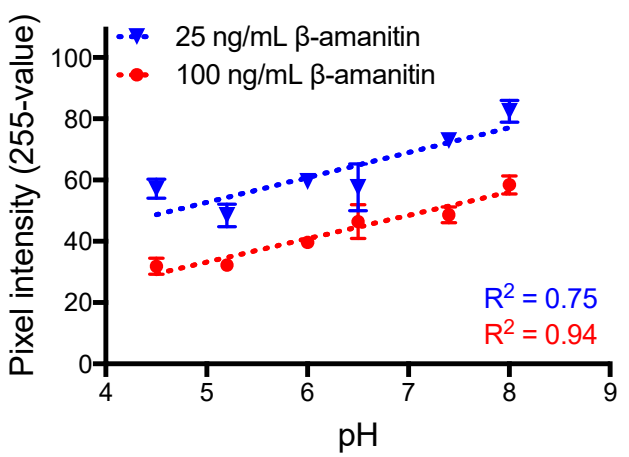

Figure 3. Test line intensities of the LFIA for solutions of $\beta$-amanitin in phosphate-buffered saline at different pHs.

\subsection{Detection of Amatoxins in Blind Fortified Human Urine Samples}

We recapitulated a human exposure study by spiking unexposed human urine samples with concentrations ( 45 to $4550 \mathrm{ng} / \mathrm{mL}$ ) of amatoxins measured from actual food exposures [12]. Two modifications were made to the sample set: (1) we included $\gamma$-AMA along with $\alpha$-AMA and $\beta$-AMA, and (2) we utilized a mixture of single and pooled urine samples. LFIA analysis was conducted as a blind test so that the LFIA readers would utilize only the LFIA as the detection method to see how well they could identify amatoxin-containing urine samples. All samples were also validated using an LC-MS/MS method [17] for confirmation. Figure 4 shows the nominal spiked concentrations along with LFIA and LC-MS/MS results.

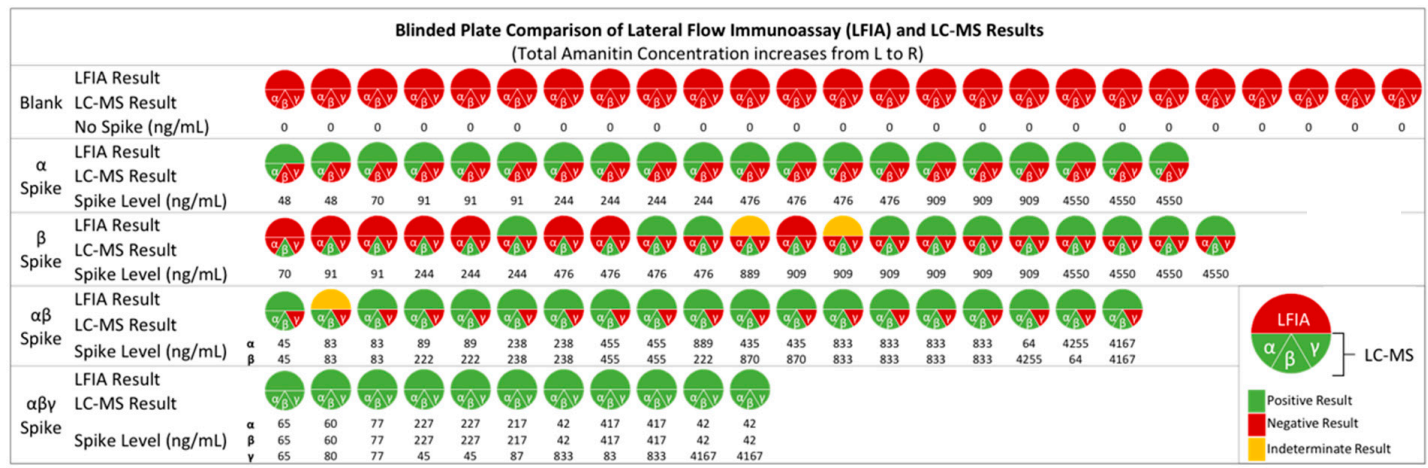

Figure 4. Design and results of experiments on amatoxin-fortified human urine samples. The toxin concentrations shown are the nominal spiked amounts. LC-MS: liquid chromatography-mass spectrometry, L: left, R: right.

In this sample set, the diagnostic efficiency of the LFIA for indicating when a sample contained amatoxins and when it did not was outstanding (94.6\%) (Table 1). Diagnostic sensitivity (true positive rate) was $92.3 \%$ and specificity (true negative rate) was 100\%. These diagnostic descriptor calculations took into account the cut-off values determined for each analyte (i.e., $10 \mathrm{ng} / \mathrm{mL}$ for $\alpha$-AMA and $\gamma$-AMA, and $100 \mathrm{ng} / \mathrm{mL}$ for $\beta$-AMA). Overall, there were only three samples that were recorded as indeterminate due to the difference in interpretation by two independent readers.

Using the cut-off values for each analyte compared to the nominal spike value resulted in a designation of "false negative" for only five samples. A false negative result meant that the LFIA reported a negative result, although the nominal concentration was greater than the cut-off value that should have resulted in a positive detection result. All five false negative designations occurred in samples that contained only $\beta$-AMA in the amount of $244-909 \mathrm{ng} / \mathrm{mL}$ (Figure 4). Of these five incorrectly identified samples, three were from single urine samples, while the other two were from 
the pooled urine matrix. This error is plausibly due to the interpretation of a faint test line as the toxin concentration approaches the cut-off value of the assay.

Interestingly, 7 samples fortified with only $\beta$-AMA in the range of $244-909 \mathrm{ng} / \mathrm{mL}$ tested positive and were reported as true positive, since they matched the criteria of being above the assay's cut-off value. All the other samples fortified with only $\beta$-AMA below this range (i.e., $91 \mathrm{ng} / \mathrm{mL}$ or lower; $n=3$ ) tested negative, and the samples that were above this range (i.e., $4550 \mathrm{ng} / \mathrm{mL} ; n=4$ ) tested positive, as expected. Overall, inaccuracy of LFIA results is observed when only $\beta$-AMA is detected at a level close to its cut-off value. For $\alpha$-AMA and $\gamma$-AMA, the concentrations tested were all at least 4-fold higher than the cut-off value, since these concentrations were those that were previously reported for amatoxin poisoning cases, and thus are clinically relevant. Detecting low concentrations of $\beta$-AMA may appear to be a limitation of this technology; however, $\alpha$-AMA and $\beta$-AMA are almost always found at comparable concentrations in mushrooms [27,28], and thus either analyte serves as a good biomarker for determining amatoxin poisoning.

Table 1. Performance of LFIA for qualitative determination of the presence of amatoxins in blind fortified urine samples and collected dog urine samples.

\begin{tabular}{ccc}
\hline Diagnostic Parameter & Fortified Human Urine $^{\mathbf{a}}$ & Intoxicated Dog Urine $^{\mathbf{b}}$ \\
\hline \# of samples & $n=96^{\mathrm{c}}$ & $n=38$ \\
\hline True positive (TP) & 60 & 8 \\
True negative (TN) & 28 & 22 \\
False positive (FP) & 0 & 0 \\
False negative (FN) & 5 & 8 \\
\hline Sensitivity & $92.3 \%$ & $50 \%$ \\
Specificity & $100 \%$ & $100 \%$ \\
Efficiency & $94.6 \%$ & $78.9 \%$
\end{tabular}

a Compared to the LC-MS/MS method [17] for confirmation. ${ }^{b}$ Compared to LC-MS/MS/MS method [20] for confirmation. ${ }^{c} 3$ samples were not included in this analysis, because the LFIA results obtained by two independent readers were ambiguous.

Most previous amatoxin exposure studies measured $\alpha$-AMA and sometimes $\beta$-AMA, however $\gamma$-AMA was often not included. Although the kinetics of $\gamma$-AMA are not well studied, we sought to include it because it could conceivably be a diagnostic marker of amatoxin poisoning. The previously described studies relying on immunoanalytical methods might also have been detecting $\gamma$-AMA, because their reagents cross-reacted with this analyte [25]. Furthermore, the previously described immunoanalytical methods were less sensitive to $\beta$-AMA [29], thus those tests would have also missed the samples that this LFIA missed when only $\beta$-AMA was present in a urine sample.

The use of pooled and single urine samples was meant as a means to identify any potentially interfering components found in urine. In the instances of inaccuracy mentioned above, no differences were attributed to those samples being a single or a pooled urine sample. Furthermore, there were no false positives observed in this amatoxin-fortified human urine study, which means that when a sample had no amatoxins below the defined threshold concentration, it was interpreted correctly by the LFIA. Together, these results underscore that no apparent urine components (natural or potentially synthetic - urine samples were neither subjected to drug screening nor were they deemed free of drugs) interfere with amatoxin detectability.

\subsection{Detection of Amatoxins in Dog Urine Samples}

Unfortunately, many dogs each year are poisoned by amatoxins due to their natural curiosity and indiscriminate eating habits [30,31]. To evaluate this LFIA for diagnostic potential, we collaboratively analyzed samples submitted to the California Animal Health and Food Safety Laboratory in Davis, CA, USA (CAHFS Davis). Urine samples were qualitatively analyzed both by LFIA and LC-MS/MS/MS methods [20] (Table 1). These samples were collected from the dogs presumed to have ingested 
amatoxin-containing mushrooms and from the dogs not suspected of mushroom poisoning (a mix of healthy and sick dogs).

The LC-MS/MS/MS method only detects $\alpha$-AMA. The LC-MS/MS/MS result reports "positive" if the sample has detectable (above $1 \mathrm{ng} / \mathrm{mL}$ ) amounts of $\alpha$-AMA, reports "negative" when no amounts are detected, and reports "trace" when a feature is detected with the correct retention time, molecular weight, and fragmentation pattern, but the concentration is below $1 \mathrm{ng} / \mathrm{mL}$.

As shown in Figure 5, the LFIA should and does indicate negative when the LC-MS/MS/MS indicates trace $(n=7)$, since the limit of detection for the LC-MS/MS/MS is $1 \mathrm{ng} / \mathrm{mL}$, which is 10 -fold lower than for the LFIA. Only one sample was found to be negative by the LFIA and positive by the LC-MS/MS/MS. This particular sample had an estimated LC-MS/MS/MS concentration of $2 \mathrm{ng} / \mathrm{mL}$, which is below the LFIA's cut-off limit $(10 \mathrm{ng} / \mathrm{mL})$, but above the threshold for positive for the LC-MS/MS/MS (1 ng/mL). However, for the rest of the samples that had either detectable amounts of $\alpha$-AMA as determined by the LC-MS/MS/MS (i.e., true positive, $n=8$ ) or non-detectable amounts of $\alpha$-AMA as determined by the LC-MS/MS/MS (i.e., true negative, $n=22$ ), the LFIA correlates $100 \%$ (Table 1). Based on this consistency, there seems to be no components in dog urine that interfere with generating reliable results. Based on this dog urine sample set, the calculated values for diagnostic sensitivity $(50 \%)$, specificity $(100 \%)$, and efficiency $(78.9 \%)$ are provided in Table 1 . This sample set distribution is not the representative distribution of prevalence of the poisonings encountered in the population, and so a larger sample size would help to determine diagnostic characteristics more accurately.

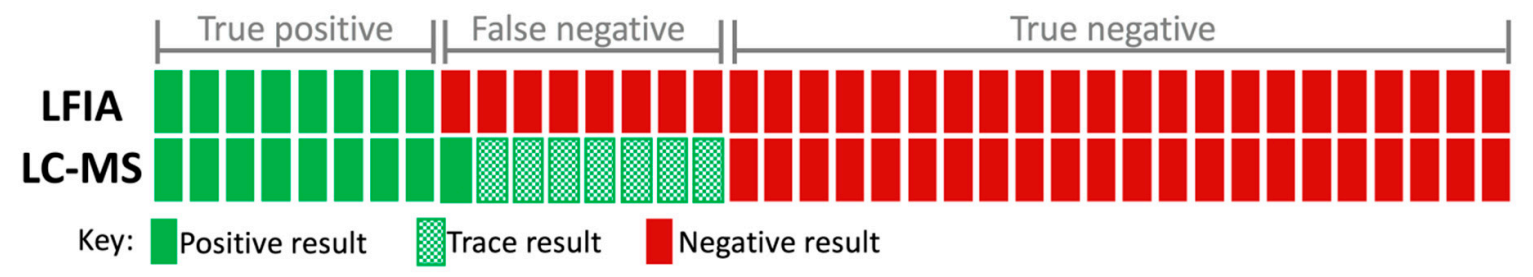

Figure 5. Comparison of methods (LC-MS/MS/MS and LFIA) for determining the presence of amatoxins in intoxicated dog urine samples $(n=38)$.

All detection methods have their benefits and limitations. LC-MS methods can provide a more definitive analysis for the samples containing lower concentrations of amatoxins, but all samples (e.g., urine and mushrooms) require sample extraction before detection. This LFIA method is exceptionally rapid (10 $\mathrm{min})$, requires no sample extraction for urine, and the test is portable. In the mushroom poisoning scenarios where the illness progresses rapidly, the LFIA described here is distinctively appropriate for point-of-care urine testing. The speed of analysis and lack of requirement for trained personnel and expensive instrumentation make this an ideal point-of-care method. Because there is no clinical tool to determine amatoxin poisoning, this LFIA test should be further exploited given its reliable diagnostic performance in this study.

\section{Materials and Methods}

\subsection{Materials and Approvals}

All the unexposed human urine samples were obtained from a commercial provider (Tennessee Blood Services, Memphis, TN, USA) and pre-screened by the vendor in accordance with FDA regulations, and thus no consent procedures were required for this project (IRB \#201210385). All animal urine samples were collected and submitted by the owners with their consent or by their veterinarians to the California Animal Health and Food Safety Laboratory System, Davis, and thus did not require the Institutional Animal Care and Use Committee review.

Monoclonal antibody (AMA9G3), hapten-protein conjugate (LB-AMA-BSA), and full LFIA test strips were produced as described earlier $[26,32,33]$. The LFIA components are diagramed in Figure 6. 
The standards used were $\alpha$-amanitin ( $\alpha$-AMA; $\geq 90 \%$, Enzo Life Sciences, Farmingdale, NY, USA), $\beta$-AMA ( $\geq 90 \%$, Enzo), and $\gamma$-AMA ( $\geq 90 \%$, Enzo).

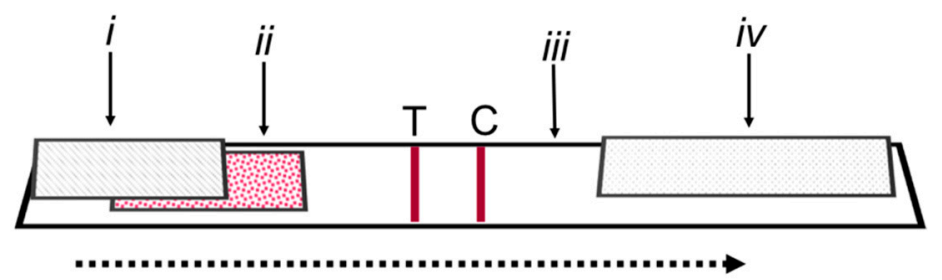

Figure 6. Schematic diagram of the lateral flow strip components: (i) sample pad, (ii) conjugate pad, (iii) nitrocellulose membrane, (iv) wicking pad, (T) test line, (C) control line. The arrow indicates the flow direction.

\subsection{Analytical Sensitivity of the LFIA}

The sensitivity of the LFIA for $\alpha$-AMA, $\beta$-AMA, and $\gamma$-AMA was determined using a pooled urine matrix. The urine matrix for calibration curves was generated using 10 single urine samples pooled together. All 10 single urine samples were tested separately as negative controls and confirmed to not interfere with the read-out. Two-fold dilutions of the standards ranging from 0.08 to $2000 \mathrm{ng} / \mathrm{mL}$ were made using the pooled urine as the diluent. Then, $100 \mu \mathrm{L}$ of each concentration were applied to the sample pad region of the test strip and tested in triplicate. The signal intensity of both the control and test lines was resolved in $10 \mathrm{~min}$. If no visible control line appeared on the test strip, the test was determined to be invalid. Digital photographs of the test strips were obtained using a Nikon SLR camera equipped with an LED ring light (B\&H Foto and Electronics Corps, New York, NY, USA) for even lighting. The images were analyzed using ImageJ software (NIH, Bethesda, MD, USA). Images were contrast-enhanced (default setting of $0.3 \%$ ), and boxes of consistent size were used to integrate the test line's pixel value. Pixel values were inverted by subtracting the measured value from the maximum possible (i.e., 255). Data was plotted using GraphPad Prism 7 software (GraphPad Software, San Diego, CA, USA) using a four-parameter logistic equation.

PBS solutions ( $10 \mathrm{mM}$ phosphate, $138 \mathrm{mM} \mathrm{NaCl}, 2.7 \mathrm{mM} \mathrm{KCl}, \mathrm{pH} 7.4$ ) were adjusted to $\mathrm{pH} 4.5$, $5.2,6,6.5,7.4$, and 8 by adding either $1 \mathrm{M} \mathrm{NaOH}$ or $1 \mathrm{M} \mathrm{HCl}$. Each solution was then spiked with $\beta$-AMA at 100 and $25 \mathrm{ng} / \mathrm{mL}$. As described above, $100 \mu \mathrm{L}$ of each solution were applied to the sample pad region of the test strip, tested in triplicate, incubated for $10 \mathrm{~min}$, photographed, and interpreted by digital analysis.

\subsection{Amatoxin-Fortified Human Urine Analysis}

Single $(n=48)$ and pooled $(n=48)$ human urine samples (obtained under IRB \#201210385) were fortified (blind to the LFIA researchers) with varying amounts of $\alpha$-AMA, $\beta$-AMA, and $\gamma$-AMA. A 96-well microtiter plate was prepared by spiking urine with $\alpha$-AMA, $\beta$-AMA, and $\gamma$-AMA at concentrations ranging from 45 to $4550 \mathrm{ng} / \mathrm{mL}$ following earlier findings from exposed individuals [12]. The distribution of samples was intended to follow these findings such that 19 wells contained $\alpha$-AMA and $\beta$-AMA, 20 wells contained only $\alpha$-AMA, 21 wells contained only $\beta$-AMA, 11 wells contained $\alpha$-AMA, $\beta$-AMA, and $\gamma$-AMA, and 25 wells contained no amatoxins. These spiked concentrations were randomly distributed between the single and pooled urine matrices.

For analytical confirmation, all human urine samples were also analyzed following the previously validated LC-MS/MS method used for human urine [17]. The LFIA method was performed by placing $100 \mu \mathrm{L}$ of a urine sample directly onto the sample pad and waiting for approximately $10 \mathrm{~min}$ before interpreting the line intensity of the test line. A visual qualitative reading of either $\mathrm{YES}(+)$ or $\mathrm{NO}(-)$ (YES - no visible test line, $\mathrm{NO}$ - a visible test line) was performed by two individuals, and a digital image of the strip was also acquired. If no control line appeared, then the test was determined to be invalid. The LFIA's cut-off values were set at $10 \mathrm{ng} / \mathrm{mL}$ for $\alpha$-AMA and $\gamma$-AMA, and at $100 \mathrm{ng} / \mathrm{mL}$ for 
$\beta$-AMA. If amatoxin concentrations exceeded these values, the result was to read positive (defined as true positive), and if the amatoxin concentrations were below these cut-off values, the result was to read negative (defined as true negative). On the basis of these studies, diagnostic sensitivity (true positive rate), diagnostic specificity (true negative rate), and diagnostic efficiency of the LFIA results were calculated using the following formulae:

$$
\begin{aligned}
\text { False positive }(\mathrm{FP})= & \text { LFIA positive, although the amatoxin concentration } \\
& \text { was below the cut-off value } \\
\text { False negative }(\mathrm{FN})= & \text { LFIA negative, although the amatoxin concentration } \\
& \text { was above the cut-off value } \\
\text { Diagnostic sensitivity }= & \mathrm{TP} /(\mathrm{TP}+\mathrm{FN}) \\
\text { Diagnostic specificity }= & \mathrm{TN} /(\mathrm{TN}+\mathrm{FP}) \\
\text { Single-study diagnostic efficiency }= & (\mathrm{TP}+\mathrm{TN}) /(\mathrm{TP}
\end{aligned}
$$

\subsection{Collected Dog Urine Analysis for Amatoxins}

Urine samples of the dogs with suspected amatoxin intoxication were submitted by their owners or by their veterinarians. For analytical confirmation, dog urine samples were analyzed following the previously validated LC-MS/MS/MS method used for dog urine [20]. The LFIA method was performed as described previously for human urine samples by placing $100 \mu \mathrm{L}$ of a urine sample directly onto the sample pad and waiting for approximately $10 \mathrm{~min}$ before interpreting the line intensity of the test line.

Author Contributions: C.S.B., K.D.S., E.I.H., and L.H.S. designed research; C.S.B., M.F., K.D.S., and E.I.H. performed research; R.H.P., M.F., K.D.S., E.I.H., and J.K. contributed samples and analytic tools; C.S.B., K.D.S., E.I.H., M.F., L.W.C., and L.H.S. analyzed data; C.S.B. wrote the original draft; J.K., R.H.P., M.F., K.D.S., E.I.H., L.W.C., and L.H.S. reviewed and edited the manuscript. All authors have read and agreed to the published version of the manuscript.

Funding: This research was funded by the United States Department of Agriculture, Agricultural Research Service, National Program project NP108, CRIS 2030-42000-049-00D, and the USDA's Innovation Fund Award.

Acknowledgments: We thank Gary Richter and Mike Barlia for supplying dog urine specimens. Many thanks to Thomas Henderson, Paula Do, and Paul Merrill for their technical assistance.

Conflicts of Interest: The authors declare no conflict of interest.

Disclaimer: The findings and conclusions in this study are those of the authors and do not necessarily represent the views of the U.S. Department of Health and Human Services, or the U.S. Centers for Disease Control and Prevention. Use of trade names and commercial sources is for identification only and does not constitute endorsement by the U.S. Department of Health and Human Services, or the U.S. Centers for Disease Control and Prevention.

\section{References}

1. Brandenburg, W.E.; Ward, K.J. Mushroom poisoning epidemiology in the United States. Mycologia 2018, 110, 637-641. [CrossRef] [PubMed]

2. Walton, J.D. The Cyclic Peptide Toxins of Amanita and Other Poisonous Mushrooms, 1st ed.; Springer Science \& Business Media: New York, NY, USA, 2018.

3. Kaya, E.; Karahan, S.; Bayram, R.; Yaykasli, K.O.; Colakoglu, S.; Saritas, A. Amatoxin and phallotoxin concentration in Amanita phalloides spores and tissues. Toxicol. Ind. Health 2015, 31, 1172-1177. [CrossRef] [PubMed]

4. Yilmaz, I.; Ermis, F.; Akata, I.; Kaya, E. A case study: What doses of Amanita phalloides and amatoxins are lethal to humans? Wilderness Environ. Med. 2015, 26, 491-496. [CrossRef]

5. Graeme, K.A. Mycetism: A review of the recent literature. J. Med. Toxicol. 2014, 10, 173-189. [CrossRef] [PubMed]

6. Benjamin, D.R. Mushrooms: Poisons and Panaceas: A Handbook for Naturalists, Mycologists, and Physicians; W. H. Freeman and Company: New York, NY, USA, 1995; p. 422.

7. Li, Y.; Mu, M.Y.; Yuan, L.; Zeng, B.M.; Lin, S.D. Challenges in the early diagnosis of patients with acute liver failure induced by amatoxin poisoning two case reports. Medicine 2018, 97, e11288. [CrossRef] [PubMed]

8. Costantino, D. Mushroom poisoning in Italy. Int. J. Artif Organs 1978, 1, 257-259. [CrossRef] 
9. Diaz, J.H. Amatoxin-containing mushroom poisonings: Species, toxidromes, treatments, and outcomes. Wilderness Environ. Med. 2018, 29, 111-118. [CrossRef]

10. Koksal, O.; Armagan, E.; Inal, T.; Ayyildiz, T.; Oner, N. A 4 year retrospective analysis of our patients with mushroom poisoning. Hong Kong J. Emerg. Med. 2013, 20, 105-110. [CrossRef]

11. Ward, J.; Kapadia, K.; Brush, E.; Salhanick, S.D. Amatoxin poisoning: Case reports and review of current therapies. J. Emerg. Med. 2013, 44, 116-121. [CrossRef]

12. Jaeger, A.; Jehl, F.; Flesch, F.; Sauder, P.; Kopferschmitt, J. Kinetics of amatoxins in human poisoning-Therapeutic implications. J. Toxicol. Clin. Toxicol. 1993, 31, 63-80. [CrossRef]

13. Butera, R.; Locatelli, C.; Coccini, T.; Manzo, L. Diagnostic accuracy of urinary amanitin in suspected mushroom poisoning: A pilot study. J. Toxicol. Clin. Toxicol. 2004, 42, 901-912. [CrossRef] [PubMed]

14. Sun, J.; Niu, Y.M.; Zhang, Y.T.; Li, H.J.; Yin, Y.; Zhang, Y.Z.; Ma, P.B.; Zhou, J.; Huang, L.; Zhang, H.S.; et al. Toxicity and toxicokinetics of Amanita exitialis in beagle dogs. Toxicon 2018, 143, 59-67. [CrossRef] [PubMed]

15. Morel, S.; Fons, F.; Rapior, S.; Dubois, V.; Vitou, M.; Portet, K.; Dore, J.-C.; Poucheret, P. Decision-making for the detection of amatoxin poisoning: A comparative study of standard analytical methods. Cryptogam. Mycol. 2016, 37, 217-239. [CrossRef]

16. Broussard, C.N.; Aggarwal, A.; Lacey, S.R.; Post, A.B.; Gramlich, T.; Henderson, J.M.; Younossi, Z.M. Mushroom poisoning-From diarrhea to liver transplantation. Am. J. Gastroenterol. 2001, 96, 3195-3198. [PubMed]

17. Abbott, N.L.; Hill, K.L.; Garrett, A.; Carter, M.D.; Hamelin, E.I.; Johnson, R.C. Detection of alpha-, beta, and gamma-amanitin in urine by LC-MS/MS using ${ }^{15} \mathrm{~N}_{10}$-alpha-amanitin as the internal standard. Toxicon 2018, 152, 71-77. [CrossRef]

18. Zhang, S.; Zhao, Y.F.; Li, H.J.; Zhou, S.; Chen, D.W.; Zhang, Y.Z.; Yao, Q.M.; Sun, C.Y. A simple and high-throughput analysis of amatoxins and phallotoxins in human plasma, serum and urine using UPLC-MS/MS combined with prime HLB elution platform. Toxins 2016, 8, 128. [CrossRef]

19. Leite, M.; Freitas, A.; Azul, A.M.; Barbosa, J.; Costa, S.; Ramos, F. Development, optimization and application of an analytical methodology by ultra performance liquid chromatography-tandem mass spectrometry for determination of amanitins in urine and liver samples. Anal. Chim. Acta 2013, 799, 77-87. [CrossRef]

20. Filigenzi, M.S.; Poppenga, R.H.; Tiwary, A.K.; Puschner, B. Determination of alpha-amanitin in serum and liver by multistage linear ion trap mass spectrometry. J. Agric. Food Chem. 2007, 55, 2784-2790. [CrossRef]

21. Maurer, H.H.; Schmitt, C.J.; Weber, A.A.; Kraemer, T. Validated electrospray liquid chromatographic-mass spectrometric assay for the determination of the mushroom toxins alpha- and beta-amanitin in urine after immunoaffinity extraction. J. Chromatogr. B 2000, 748, 125-135. [CrossRef]

22. Gicquel, T.; Lepage, S.; Fradin, M.; Tribut, O.; Duretz, B.; Morel, I. Amatoxins (alpha- and beta-amanitin) and phallotoxin (phalloidin) analyses in urines using high-resolution accurate mass LC-MS technology. J. Anal. Toxicol. 2014, 38, 335-340. [CrossRef]

23. Defendenti, C.; Bonacina, E.; Mauroni, M.; Gelosa, L. Validation of a high performance liquid chromatographic method for alpha amanitin determination in urine. Forensic Sci. Int. 1998, 92, 59-68. [CrossRef]

24. Abuknesha, R.A.; Maragkou, A. A highly sensitive and specific enzyme immunoassay for detection of beta-amanitin in biological fluids. Anal. Bioanal. Chem 2004, 379, 853-860. [CrossRef] [PubMed]

25. Bühlmann Laboratories, A.G. Amanitin ELISA Product Insert; Catalog\# ek-am1; Bühlmann Laboratories AG: Schönenbuch, Switzerland, 2013.

26. Bever, C.S.; Adams, C.A.; Hnasko, R.M.; Cheng, L.W.; Stanker, L.H. Development and application of a lateral flow immunoassay for the rapid and sensitive detection of lethal amatoxins from mushrooms. Toxins 2020. under review.

27. Enjalbert, F.; Gallion, C.; Jehl, F.; Monteil, H.; Faulstich, H. Amatoxins and phallotoxins in Amanita species-high-performance liquid-chromatographic determination. Mycologia 1993, 85, 579-584. [CrossRef]

28. Yilmaz, I.; Kaya, E.; Sinirlioglu, Z.A.; Bayram, R.; Surmen, M.G.; Colakoglu, S. Clinical importance of toxin concentration in Amanita verna mushroom. Toxicon 2014, 87, 68-75. [CrossRef] [PubMed]

29. Staack, R.F.; Maurer, H.H. New Bühlmann ELISA for determination of amanitins in urine-Are there false positive results due to interferences with urine matrix, drugs or their metabolites? Toxichem. Krimtech. 2000, 68, 68-71.

30. Cope, R.B. Mushroom poisoning in dogs. Vet. Med. 2007, 102, 95-100. 
31. Puschner, B.; Wegenast, C. Mushroom poisoning cases in dogs and cats: Diagnosis and treatment of hepatotoxic, neurotoxic, gastroenterotoxic, nephrotoxic, and muscarinic mushrooms. Vet. Clin. North Am. Small Anim. Pract. 2018, 48, 1053-1067. [CrossRef]

32. Bever, C.S.; Barnych, B.; Hnasko, R.; Cheng, L.W.; Stanker, L.H. A new conjugation method used for the development of an immunoassay for the detection of amanitin, a deadly mushroom toxin. Toxins 2018, 10, 265. [CrossRef]

33. Bever, C.S.; Hnasko, R.M.; Cheng, L.W.; Stanker, L.H. A rapid extraction method combined with a monoclonal antibody-based immunoassay for the detection of amatoxins. Toxins 2019, 11, 724. [CrossRef]

(C) 2020 by the authors. Licensee MDPI, Basel, Switzerland. This article is an open access article distributed under the terms and conditions of the Creative Commons Attribution (CC BY) license (http://creativecommons.org/licenses/by/4.0/). 